\title{
Cognitive Consequences in Children with Epilepsy
}

\author{
Hideaki Kanemura ${ }^{a, *}$ and Masao Aihara ${ }^{\mathrm{b}}$
}

a Department of Pediatrics, Faculty of Medicine, University of Yamanashi, 1110 Chuo, Yamanashi 409-3898,
Japan
${ }^{b}$ Interdisciplinary Graduate School of Medicine and Engineering, University of Yamanashi, 1110 Chuo,
yamanashi 409-3898, Japan

\begin{abstract}
Some epilepsy is, in general, often associated with cognitive problems that can also affect a patient's adjustments. Epileptic seizures result from an excessive, synchronous discharge of cerebral neurons. Interictal paroxysmal electroencephalogram (EEG) abnormalities are regarded as a correlate of persistent pathological neuronal discharges. Thus, correlation between cognitive deterioration and seizure severities/EEG paroxysmal abnormalities should be investigated. We have previously measured frontal/prefrontal lobe volumes using three-dimensional (3D)magnetic resonance imaging (MRI) in children with benign childhood epilepsy with centrotemporal spikes, epilepsy with continuous spike-waves during slow sleep, frontal lobe epilepsy, and Panayiotopoulos syndrome, and confirmed that longer active seizure period as frequent spike-waves coupled with the occurrence of frequent seizures and presence of status epilepticus may be associated with prefrontal lobe growth disturbance, which relates to cognitive impairments. These findings suggest that seizure severities such as repeated seizures and presence of status epilepticus, and the subclinical paroxysmal EEG abnormalities may induce prefrontal lobe growth disturbance, which leads to intellectual impairments. Achieving better seizure control and remission for paroxysmal EEG abnormalities is a key to improve quality of life (QOL) in children with epilepsy. From the perspective of decreased cognitive problems and improving QOL, management may be required to remit seizures and paroxysmal EEG abnormalities as soon as possible to achieve optimal prognosis in epilepsy.
\end{abstract}

Keywords: Epilepsy, Seizure severity, Electroencephalogram (EEG), Intellectual impairments, Children.

\section{INTRODUCTION: COGNITIVE ASPECTS IN CHILDREN WITH EPILEPSY}

Suffering from epilepsy is not limited to having seizures. Some epilepsy is, in general, often associated with cognitive problems that can also affect a patient's adjustments. In children with epilepsy syndromes such as frontal lobe epilepsy (FLE) or epilepsy with continuous spike-waves during slow sleep (CSWS), one or more cognitive functions were deteriorated in several degree [1-3]. Even in the benign epilepsies with an excellent prognosis such as benign childhood epilepsy with centrotemporal spikes (BCECTS) or Panayiotopoulos syndrome (PS), some children with these epilepsies can demonstrate intellectual impairments $[4,5]$. The neuropsychologic deficits cover a wide range of brain-behavior domains, including attention, memory, mood, language, visuospatial and executive functions, intelligence, and social skills. The relevance of these subtle dysfunctions as well as their correlation with seizures or interictal electroencephalogram (EEG) abnormalities is unclear.

Epileptic seizures result from an excessive synchronous discharge of cerebral neurons. On the other hand, interictal paroxysmal EEG abnormalities

*Address correspondence to this author at the Department of Pediatrics, Faculty of Medicine, University of Yamanashi, 1110 Shimokato, Chuo-city, Yamanashi 409-3898, Japan; Tel: +81-55-273-9606; Fax: +81-55-273-6745; E-mail: ykimu@yamanashi.ac.jp are regarded as a correlate of persistent pathological neuronal discharges [6]. Accordingly, these clinical and EEG markers should be sought to predict poor cognitive outcome.

Children and adolescents with epileptic abnormalities involving the frontal lobe show significant impairment of executive abilities (abilities primarily processed by the frontal lobe), which can relate to intellectual impairments [7]. EEG monitoring can detect subclinical paroxysmal abnormalities, which may affect attention and other aspects of cognitive functioning in various ways, even in the absence of clinical seizures. In addition, seizure severities such as seizure frequency and presence of status epilepticus (SE) may also affect cognitive deterioration in children with epilepsy. Thus, correlation between cognitive deterioration and seizure severities/EEG paroxysmal abnormalities should be investigated.

\section{METHODS}

We registered patients with BCECTS, PS, FLE, and CSWS at our hospital. Serial three-dimensional (3D)magnetic resonance imaging (MRI) studies were performed in these patients. The 3D-MRI data were acquired according to our published method, in briefly described as follows [8]. The 3D-MRI data were acquired using a fast spoiled gradient recalled echo in steady-state with a 3D Fourier transformation 
sequence, and $3 \mathrm{D}$ images of the whole brain surface were obtained from the 124 sections using an Advantage Windows RP 3D Analyzer workstation (General Electric, Wisconsin, MW, USA). Thereafter, the frontal lobe was identified and further analyzed using our published method [8]. The central and precentral sulci were identified in left and right lateral as well as superior 3D views of the brain surface. The frontal and prefrontal lobes were defined as the regions anterior to the central and precentral sulcus, respectively. We also confirmed the extent of the frontal and prefrontal lobes by comparing scans with images resliced in the axial plane parallel to the anterior and posterior commissure line. Finally, we measured frontal and prefrontal lobe volumes using the volume measurement function of the workstation and the 3D image data. A control group neurologically normal age-matched children was also examined using MRI.

\section{RESULTS}

\section{Studies in BCECTS Group}

In our previous study [4], serial changes in frontal and prefrontal lobe volumes using 3D MRI were measured in children with BCECTS with (BCECTS $(+)$ ) or without (BCECTS (-)) cognitive impairments. In addition, we evaluated correlations between prefrontal lobe growth and active seizure period. In this study, frontal and prefrontal lobes revealed growth disturbance in BCECTS (+) compared with BCECTS (-) and control subjects. Moreover, prefrontal-to-frontal lobe volume ratio increased serially in BCECTS (-) similarly to controls, but was stagnant or decreased in BCECTS (+). These findings suggest that longer active seizure period as frequent spike-waves coupled with the occurrence of frequent seizures may be associated with prefrontal lobe growth disturbance, which relates to cognitive impairments. In another study, the cognitive function of BCECTS (-) participants was within the normal range, whereas BCECTS (+) participants exhibited cognitive deterioration 1 year after onset and persistent cognitive impairments for several years. The results of this study showed that a combination of spike rate and extended periods of high-frequency paroxysmal EEG abnormalities may predict the evolution of BCECTS (+) [4].

\section{Study in PS Group}

PS is usually not associated with cognitive impairments. However, intellectual impairments may also be present in at least some of the patients with
PS. In our previous study [5], we prospectively measured frontal and prefrontal lobe volumes using 3D MRI in patients with PS with and without SE. In addition, serial changes in Wechsler intelligence scale for children (WISC) average scores were also evaluated. This study showed that growth of the frontal and prefrontal lobes was slightly decreased for some time after SE episodes in the SE patients. Moreover, the prefrontal-to-frontal lobe volume ratio was stagnant for some time after SE in the SE patients. On the other hand, the average scores of full intelligent quotient (FIQ) in SE patients showed a reduction 2 years after seizure onset. In particular, the average scores of performance intelligent quotient (PIQ) revealed a predominant reduction 2 years after seizure onset. These scores in SE patients did not reach the levels of the initial examinations. These findings came up with the conclusion that occurrence of SE in patients with PS at least in some patients may be associated with retarded prefrontal lobe growth, which was related to intellectual impairments [5].

\section{Study in FLE Group}

Children with FLE manifest significant psychosocial problems including intellectual impairments relative to normative standards. Recent articles have reached the conclusion that seizures impair the developing brain. Accordingly, repeated seizures may lead to cognitive impairments. In our previous study [1], serial changes in regional cerebral volumes were measured in FLE patients presenting with and without intractable clinical courses and cognitive impairments. This study showed that frontal and prefrontal lobes showed no obvious growth during the active seizure period in the FLE patients with intractable clinical courses and cognitive impairments. Moreover, prefrontal lobe growth also revealed more rapid recovery in a FLE patient presenting intractable clinical courses and cognitive impairments with shorter active seizure period. These findings suggest that repeated seizures may lead to prefrontal lobe growth disturbance. These findings came up with the conclusion that the occurrence of frequent seizures in patients with FLE may be associated with prefrontal lobe growth retardation, which relates to cognitive impairments [1].

\section{Study in CSWS Group}

CSWS is a rare epileptic syndrome occurring in children with severe paroxysmal EEG abnormalities and cognitive/behavioral deterioration, with or without premorbid developmental disturbances. CSWS 
manifestations strongly correlate with frontal lobe dysfunctions. In our previous volumetric studies [2, 3], frontal and prefrontal lobe volumes revealed growth disturbance in all CSWS patients compared with those of normal subjects. These studies also revealed that in patients with shorter seizure durations and CSWS periods, growth disturbance of prefrontal lobe was soon restored to a more normal growth ratio, whereas that was persistent in the patients with longer seizure durations and CSWS period. These findings came up with the conclusion that seizure and the duration of paroxysmal EEG abnormalities may be associated with prefrontal lobe growth disturbance, which relates cognitive/behavioral deterioration [2, 3].

\section{DISCUSSION}

\section{Vulnerability of Prefrontal Functions}

In the developing brain, the effects of seizures on neuronal survival and brain growth have remained controversial $[9,10]$. The immature brain appears to be more resistant to the toxic effects of glutamate than the mature brain [11]. However, several studies provide strong evidence for the vulnerability of the immature brain to seizure-induced damage, which bears features of both necrotic and apoptotic death and contributes to synaptic reorganization [12]. In addition, prefrontal lobe is among the last cortical regions to reach full structural development, therefore, prefrontal function and its disorders are not immediately apparent $[8,13]$. These findings suggest that prefrontal functions show an unusually long period of increased vulnerability, in which neurons and glial cells are readily affected by many factors including seizures and paroxysmal EEG abnormalities [1, 4, 5, 13]. Accordingly, seizure severities such as repeated seizures and presence of SE may induce prefrontal lobe growth disturbance, which leads to intellectual impairments.

\section{Correlation between Seizure Severities and Cognitive Deterioration}

More frequent and severe seizures can affect cognitive functions. Possible explanations for this relationship are summarized as follows: a) seizures and cognitive problems are associated because both are related to an underlying factor; b) seizure activity per se disrupts cognition; and c) children have a negative psychological response to seizures [14]. Several findings in our volumetric studies may support the second suggestion of Austin et al. that seizure activity per se disrupts cognition.

\section{Correlation between Paroxysmal EEG Abnormalities and Cognitive Deterioration}

Correlation between paroxysmal EEG abnormalities and cognitive impairment has been under debate. Weglage et al. concluded that spike frequency was not correlated with specific neuropsychological and language functions [15]. In contrast, it is increasingly recognized that transient cognitive impairment may accompany interictal paroxysmal EEG abnormalities, especially when these are frequent $[16,17]$. In a previous study, neuropsychological functioning improved with normalization of the EEG [18]. In addition, study by Metz-Lutz et al. showed that performances in neuropsychological assessments were specifically weakened in individuals with active epilepsy with frequent spike-wave discharges on the waking EEG [19]. These findings suggest that the subclinical paroxysmal EEG abnormalities contribute to the psychosocial problems including cognitive impairments. Interictal paroxysmal EEG abnormalities may be the major determinant of clinical features such as cognitive dysfunction [20]. The results in our previous studies may support this hypothesis.

\section{Cognitive Impairments in Benign Childhood Epilepsy}

A small proportion of cases that start out as benign childhood epilepsy such as BCECTS evolve into atypical benign partial epilepsy (ABPE) of childhood $[20,21]$. There is consensus that recognition of some atypical clinical or EEG features in cases of benign childhood epilepsy does not mean that those children are going to present severe cognitive or behavioral problems [21]. However, our current researches suggest that damage to the frontal regions during childhood due to frequent seizures and SE may interrupt normal maturational processes and organization, resulting in impairments in neurobehavioral development even in benign childhood epilepsy. Management of treatment to prevent seizures as much as possible may be required to achieve optimal prognosis in benign childhood epilepsy with atypical clinical features [4].

\section{Cognitive Impairments and Quality of Life (QOL)}

Cognitive problems can affect the QOL of children with not only epilepsy, but also other chronic illness. Just as seizures are not constant over time and are often reactive to circumstance, so too the cognitive/behavioral problems are not constant and 
reactive, as Engel et al. mentioned [22]. In contrast, a relationship between current seizure frequency and perceived stigma may be present [23]. Perception of stigma can reduce QOL. Accordingly, seizures can alter brain function in a manner that influences cognition just as cognition influences seizures. Moreover, our current study showed that a relationship was also identified between frontal EEG paroxysmal abnormality and a greater perception of stigma [24]. These findings suggest that achieving better seizure control and remission for paroxysmal EEG abnormalities is a key to improve QOL in children with epilepsy. However, although the persistence of seizures is a negative factor, rehabilitation can be used in patients with epilepsy to improve their condition [25]. Further research will be needed to confirm this point.

\section{CONCLUSION}

Clinical seizure manifestation and paroxysmal EEG abnormalities can interfere with cognitive functions. The specific nature and severity of neuropsychological problems may vary according to the particular localization and severity of seizures and EEG discharges. From the perspective of decreased cognitive problems and improving QOL, management may be required to remit seizures and paroxysmal EEG abnormalities as soon as possible to achieve optimal prognosis in epilepsy.

\section{REFERENCES}

[1] Kanemura H, Sano F, Tando T, Sugita K, Aihara M. Repeated seizures induce prefrontal growth disturbance in frontal lobe epilepsy. Brain Dev 2012; 34: 175-80. http://dx.doi.org/10.1016/j.braindev.2011.04.009

[2] Kanemura H, Sugita K, Aihara M. Prefrontal lobe growth in a patient with continuous spike-waves during slow sleep. Neuropediatrics 2009; 40: 192-4. http://dx.doi.org/10.1055/s-0029-1243224

[3] Kanemura H, Aihara M. Neurobiological effects of CSWS on brain growth: A magnetic resonance imaging volumetric study. J Pediatr Epilepsy 2012; 1: 187-93.

[4] Kanemura H, Hata S, Aoyagi K, Sugita K, Aihara M. Serial changes of prefrontal lobe growth in the patients with benign childhood epilepsy with centrotemporal spikes presenting with cognitive impairments/behavioral problems. Brain Dev 2011; 33: 106-13. http://dx.doi.org/10.1016/j.braindev.2010.03.005

[5] Kanemura H, Sano F, Ohyama T, Aoyagi K, Sugita K, Aihara $M$. Sequential prefrontal lobe volume changes and cognitive dysfunctions in children with Panayiotopoulos syndrome presenting with status epilepticus. Epilepsy Res 2015; 112: $122-9$.

http://dx.doi.org/10.1016/..eplepsyres.2015.02.019

[6] Wolff M, Weiskopf N, Serra E, Preissl H, Birbaumer N, Kraegeloh-Mann I. Benign partial epilepsy in childhood: selective cognitive deficits are related to the location of focal spikes determined by combined EEG/MEG. Epilepsia 2005; 46: 1661-7.

http://dx.doi.org/10.1111/j.1528-1167.2005.00255.x
[7] Vago C, Bulgheroni S, Franceschetti S, Riva D. Frontal lobe epilepsies: neuropsychological and behavioral consequences in children. Epilepsy in children-Clinical and social aspects. 2011; p.187-212.

[8] Kanemura H, Aihara M, Aoki S, Araki T, Nakazawa S. Development of the prefrontal lobe in infants and children: a three-dimensional magnetic resonance volumetric study. Brain Dev 2003; 25: 195-199. http://dx.doi.org/10.1016/S0387-7604(02)00214-0

[9] Camfield PR. Recurrent seizures in the developing brain are not harmful. Epilepsia 1997; 38: 735-737. http://dx.doi.org/10.1111/j.1528-1157.1997.tb01245.x

[10] Wasterlain CG. Recurrent seizures in the developing brain are harmful. Epilepsia 1997; 38: 728-734. http://dx.doi.org/10.1111/j.1528-1157.1997.tb01244.x

[11] Marks JD, Friedman JE, Haddad GG. Vulnerability of CA1 neurons to glutamate is developmentally regulated. Dev. Brain Res 1996; 97: 194-206. http://dx.doi.org/10.1016/S0165-3806(96)00149-6

[12] Sankar R, Shin D, Mazarati AM, et al. Epileptogenesis after status epilepticus reflects age- and model-dependent plasticity. Ann Neurol 2000; 48: 580-589.

http://dx.doi.org/10.1002/1531-8249(200010)48:4<580::AIDANA4>3.0.CO;2-B

[13] Dobbing J, Sands J. Vulnerability of developing brain. IX. The effect of nutritional growth retardation on the timing of the brain growth spurt. Biol Neonate 1971; 19: 363-378. http://dx.doi.org/10.1159/000240430

[14] Austin JK, Dunn DW, Caffrey HM, Perkins SM, Harezlak J, Rose DF. Recurrent seizures and behavior problems in children with first recognized seizures: a prospective study. Epilepsia 2002; 43: 1564-1573. http://dx.doi.org/10.1046/j.1528-1157.2002.26002.x

[15] Weglage J, Demsky A, Pietsch M, Kurlemann G. Neuropsychological, intellectual, and behavioral findings in patients with centrotemporal spikes with and without seizures. Dev Med Child Neurol 1997; 39: 646-651. http://dx.doi.org/10.1111/j.1469-8749.1997.tb07357.x

[16] Ong HT, Wyllie E. Benign childhood epilepsy with centrotemporal spikes: is it always benign? Neurology 2000; 54: 1182-1185.

http://dx.doi.org/10.1212/WNL.54.5.1182

[17] Fernandez IS, Loddenkemper T, Galanopoulou AS, Moshe SL. Should epileptiform discharges be treated? Epilepsia 2015; 56: 1492-1504.

http://dx.doi.org/10.1111/epi.13108

[18] Baglietto MG. Battaglia FM, Nobili L, et al. Neuropsychological disorders related to interictal epileptic discharges during sleep in benign epilepsy of childhood with centrotemporal or Rolandic spikes. Dev Med Child Neurol 2001; 43: 407-412. http://dx.doi.org/10.1017/S0012162201000755

[19] Metz-Lutz MN, Kleitz C, de Saint-Martin A, Massa R, Hirsch E, Marescaux C. Cognitive development in benign focal epilepsies of childhood. Dev Neurosci 1999; 21: 182-190. http://dx.doi.org/10.1159/000017397

[20] Kanemura H, Sano F, Aoyagi K, Sugita K, Aihara M. Do sequential EEG changes predict atypical clinical features in rolandic epilepsy? Dev Med Child Neurol 2012; 54: 912-917. http://dx.doi.org/10.1111/j.1469-8749.2012.04358.x

[21] Fejerman N. Benign childhood epilepsy with centrotemporal spikes. In: Engel J Jr, Pedley TA, editors. Epilepsy: A Comprehensive Textbook, $2^{\text {nd }}$ ed. Philadelphia, PA: Lippincott Williams \& Wilkins 2008; p. 2369-77.

[22] Engel Jr J, Taylor DC, Trimble MR. Neurobiology of behavioral disorders. Epilepsy: a comprehensive textbook. Lippincott Williams \& Wilkins. 2008, pp.2077-2083. 
[23] Kanemura H, Sano F, Sugita K, Aihara M. Presence of monthly seizures affects perceived stigma in children with epilepsy. J Pediatr Epilepsy 2014; 3: 85-92.

[24] Kanemura H, Sano F, Ohyama T, Sugita K, Aihara M. Correlation between perceived stigma and EEG paroxysmal abnormality in childhood epilepsy. Epilepsy Behav 2015; 52 : 44-48.

http://dx.doi.org/10.1016/i.yebeh.2015.08.021
[25] Chiappedi M, Beghi E, Ferrari-Ginevra O, Ghezzo A, Maggioni E, Mattana F, Spelta $P$, Stefanini MC, Biserni $P$, Tonali P. Response to rehabilitation of children and adolescents with epilepsy. Epilepsy Behav 2011; 20 79-82. http://dx.doi.org/10.1016/j.yebeh.2010.10.029 